\title{
Therapeutic Cancer Vaccination with Immunopeptidomics-Discovered Antigens Confers Protective Antitumor Efficacy
}

\author{
Karita Peltonen ${ }^{1,2,3,4}$, Sara Feola ${ }^{1,2,3,4} \mathbb{0}$, Husen M. Umer ${ }^{5}$, Jacopo Chiaro ${ }^{1,2,3,4}$, Georgios Mermelekas ${ }^{5}$, \\ Erkko Ylösmäki ${ }^{1,2,3,4}{ }^{\mathbb{D}}$, Sari Pesonen ${ }^{6}$, Rui M. M. Branca ${ }^{5}$, Janne Lehtiö ${ }^{5, *}$ and Vincenzo Cerullo ${ }^{1,2,3,4, * \mathbb{C}}$ \\ 1 Drug Research Program, Division of Pharmaceutical Biosciences, Faculty of Pharmacy, University of Helsinki, \\ 00790 Helsinki, Finland; karita.peltonen@helsinki.fi (K.P.); sara.feola@helsinki.fi (S.F.); \\ jacopo.chiaro@helsinki.fi (J.C.); erkko.ylosmaki@helsinki.fi (E.Y.) \\ 2 Helsinki Institute of Life Science (HiLIFE), University of Helsinki, 00790 Helsinki, Finland \\ 3 Translational Immunology Research Program (TRIMM), University of Helsinki, 00790 Helsinki, Finland \\ 4 iCAN Digital Precision Cancer Medicine Flagship, University of Helsinki, 00790 Helsinki, Finland \\ 5 Science for Life Laboratory, Department of Oncology-Pathology, Karolinska Institutet, 17165 Solna, Sweden; \\ husen.umer@ki.se (H.M.U.); georgios.mermelekas@ki.se (G.M.); rui.mamede-branca@ki.se (R.M.M.B.) \\ 6 Valo Therapeutics Oy, 00790 Helsinki, Finland; sari.pesonen@valotx.com \\ * Correspondence: janne.lehtio@ki.se (J.L.); vincenzo.cerullo@helsinki.fi (V.C.); Tel.: +46-8-5248-1416 (J.L.); \\ $+358-50-31-85754$ (V.C.)
}

\section{check for}

updates

Citation: Peltonen, K.; Feola, S.; Umer, H.M.; Chiaro, J.; Mermelekas, G.; Ylösmäki, E.; Pesonen, S.; Branca, R.M.M.; Lehtiö, J.; Cerullo, V. Therapeutic Cancer Vaccination with Immunopeptidomics-Discovered Antigens Confers Protective Antitumor Efficacy. Cancers 2021, 13, 3408. https://doi.org/10.3390/ cancers 13143408

Academic Editor: Antonio Marchini

Received: 3 June 2021

Accepted: 2 July 2021

Published: 7 July 2021

Publisher's Note: MDPI stays neutral with regard to jurisdictional claims in published maps and institutional affiliations.

Copyright: (c) 2021 by the authors. Licensee MDPI, Basel, Switzerland. This article is an open access article distributed under the terms and conditions of the Creative Commons Attribution (CC BY) license (https:// creativecommons.org/licenses/by/ $4.0 /)$.
Simple Summary: Immunotherapy has revolutionized cancer treatment, yet many tumors remain resistant to current immuno-oncology therapies. Here we explore a novel, customized oncolytic adenovirus vaccine platform as immunotherapy in a resistant tumor model. We present a workflow for customizing the oncolytic vaccine for improved tumor targeting. This targeting is based on experimentally discovered tumor antigens, which are incorporated as active components of the vaccine formulation. The pipeline may be further applied for designing personalized therapeutic cancer vaccines.

Abstract: Knowledge of clinically targetable tumor antigens is becoming vital for broader design and utility of therapeutic cancer vaccines. This information is obtained reliably by directly interrogating the MHC-I presented peptide ligands, the immunopeptidome, with state-of-the-art mass spectrometry. Our manuscript describes direct identification of novel tumor antigens for an aggressive triplenegative breast cancer model. Immunopeptidome profiling revealed 2481 unique antigens, among them a novel ERV antigen originating from an endogenous retrovirus element. The clinical benefit and tumor control potential of the identified tumor antigens and ERV antigen were studied in a preclinical model using two vaccine platforms and therapeutic settings. Prominent control of established tumors was achieved using an oncolytic adenovirus platform designed for flexible and specific tumor targeting, namely PeptiCRAd. Our study presents a pipeline integrating immunopeptidome analysisdriven antigen discovery with a therapeutic cancer vaccine platform for improved personalized oncolytic immunotherapy.

Keywords: tumor antigen; endogenous retrovirus; cancer vaccine; immunotherapy; immunopeptidome; ligandome; breast cancer; mass spectrometry; oncolytic vaccine

\section{Introduction}

Immunotherapy has reshaped the treatment of highly immunogenic tumors as exemplified by the success of immune checkpoint blockade for metastatic melanoma. Recent evidence implies triple-negative breast cancer (TNBC) may also be susceptible to immunotherapy: it shows high mutational load among breast cancers, expression of 
checkpoint molecules such as PD-L1, and infiltration of immune cells suggestive of preexisting immunity towards tumor. A gene expression profiling study of over 3000 cases further described an immunomodulatory subtype of TNBC enriched for genes involved in immune-related cellular processes [1]. Importantly, these immunological biomarkers associate with better clinical outcome of the patients [2,3]. In a recent phase 3 clinical trial, checkpoint inhibition in combination with paclitaxel prolonged progression-free survival of metastatic TNBC patients [4], leading to the FDA approval of the combination treatment for PD-L1-positive tumors. Despite these outstanding achievements, only a relatively select group of TNBC patients may fully benefit from the treatment. The high response rate to immunotherapy correlates with high tumor PD-L1 expression, which is present in $19 \%$ of TNBC specimens [5]. Furthermore, the tumor infiltrating lymphocytes may not target a clinically effective tumor antigen, limiting the feasibility of immune checkpoint blockade as single therapy. Combining checkpoint inhibition with targeted activation of tumor antigen-specific T-cells, as achieved with therapeutic cancer vaccination, may have the potential to broaden the applicability of checkpoint blockade therapy beyond the currently targeted patient group.

The cornerstone of effective immunotherapy is the recognition of tumor antigens by cytotoxic T-lymphocytes. On estimate, thousands of tumor antigens, short peptides displayed in the context of MHC class I molecules (MHC-I) on the tumor cell surface, can be naturally presented [6]. Knowledge of the presented tumor antigen repertoire, different from that of normal cells, is necessary for successful design of more targeted treatment modalities [7]. For several tumor types, including TNBC, information on well established, clinically relevant tumor antigens is currently limited. For tumor types with lower mutational burden, tumor-associated antigens may offer an attractive alternative to broaden the repertoire of clinically feasible targets $[7,8]$. These tumor-associated antigens are derived from either tissue-specific or overexpressed proteins in tumor, or proteins expressed in tumor due to epigenetic changes (e.g., cancer testis antigens). Furthermore, endogenous retroviruses (ERVs) are emerging as an interesting source of tumor antigens as their expression in tumor positively associates with immune cell infiltration and even with immune therapy response [9-11].

Here, we identify tumor antigens and investigate their applicability as personalized cancer vaccination in a preclinical model of TNBC. We identify the tumor-associated antigen repertoire, tumor immunopeptidome, combining MHC class-I immunoaffinity purification with state-of-the-art mass spectrometry analysis. Among the MHC-I presented immunopeptidome we discovered a novel peptide originating from an endogenous retrovirus protein. We further explored the potential of targeting selected tumor antigens using prophylactic or therapeutic vaccination in an experimental mouse model of TNBC. Our results support the notion that tumors with lower mutational burden can also be susceptible to targeted immunotherapy. Furthermore, we present a pipeline for improved cancer immunotherapy based on mass spectrometry discovery of therapeutically targetable T-cell epitopes combined with oncolytic cancer vaccines (Figure 1a). 


\section{a}

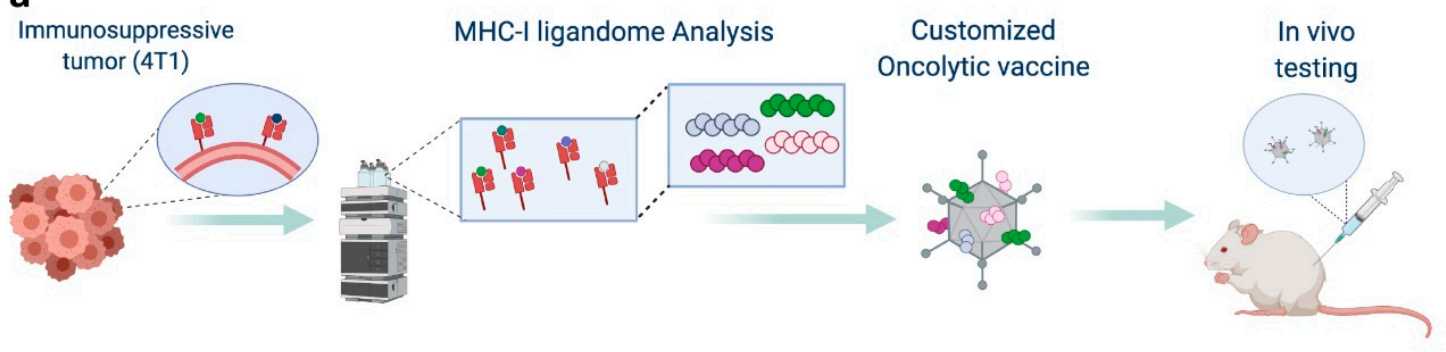

b

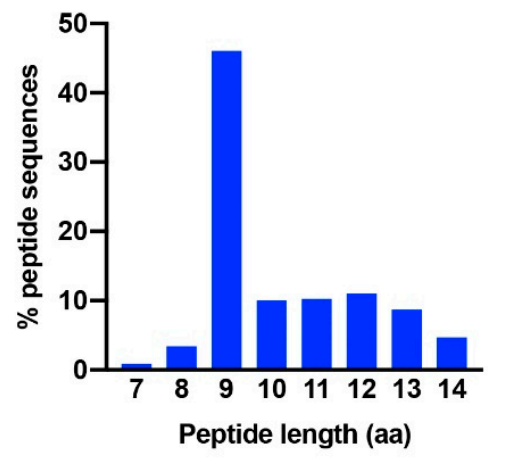

C
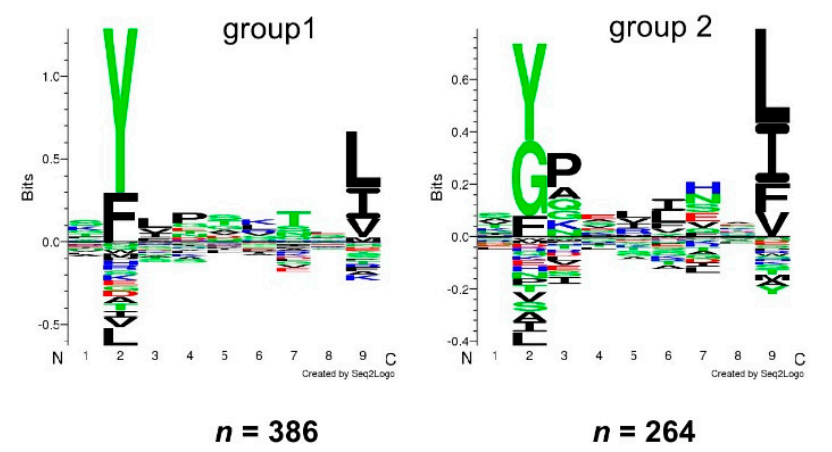

d

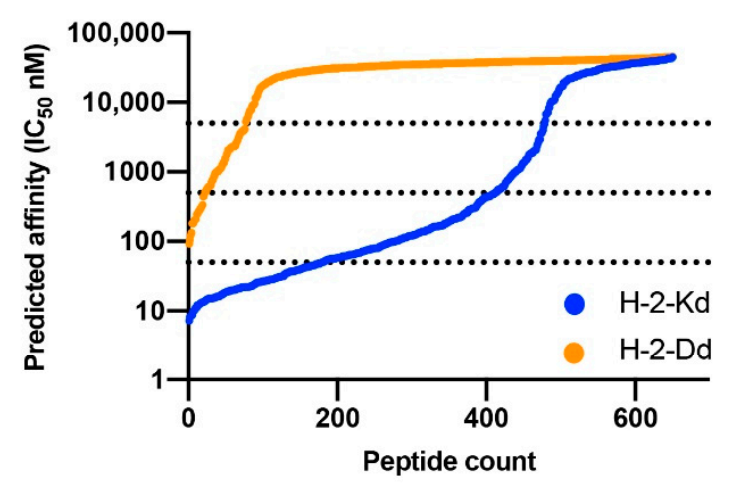

$\mathbf{e}$

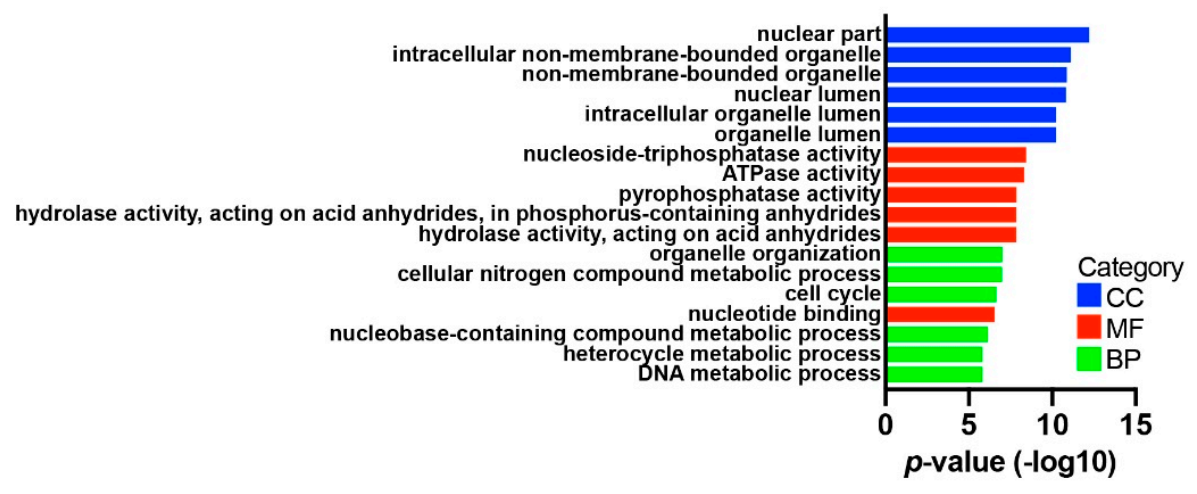

Figure 1. Characteristics of identified peptide ligands. (a) Overall workflow of the study. (b) Length distribution of the identified peptides. (c) Motif analysis of 9 mers. The 9 mers show reduced amino acid complexity at anchor positions characteristic of MHC-I peptide binding motifs expressed by the cell line. (d) MHC-I binding affinity prediction of 9 mers. Of the identified 9 mers, $67 \%$ can be assigned to $\mathrm{H}-2 \mathrm{~K}^{\mathrm{d}}$ or $\mathrm{H}-2 \mathrm{D}^{\mathrm{d}}$ and thus are considered as MHC-I ligand. (e) GO analysis of the MHC-I ligand source proteins. Most overrepresented biological processes (BP), molecular function (MF), and cellular component (CC) of the MHC-I source proteins ( $p$-values, Fisher's exact test with FDR correction) are presented. 


\section{Materials and Methods}

\subsection{Cell Lines}

As the mouse model of TNBC, we chose the $4 \mathrm{~T} 1$ mouse mammary tumor cell line, a highly metastatic TNBC type originally derived from a spontaneously arising tumor in Balb/c mice. The cell line was purchased from ATCC (Manassas, VA, USA) and cultured according to ATCC recommendations in DMEM supplemented with $10 \%$ heat-inactivated FBS and antibiotics in $+37{ }^{\circ} \mathrm{C}, 5 \% \mathrm{CO}_{2}$ in a humidified atmosphere.

\subsection{Purification of MHC Class-I Complexes}

MHC-I peptide complexes were immunoaffinity purified from the 4T1 mouse tumor cell line using MHC-I antibody against H-2Kd/H-2Dd (clone 34-1-2S, SouthernBiotech, Birmingham, AL, USA) and applying the method by Bassani-Sternberg [12] with minor modifications. Frozen 4 T1 cells $\left(1-3 \times 10^{8}\right)$ were lysed with $0.25 \%$ sodium deoxycholate, $0.2 \mathrm{mM}$ iodoacetamide, $1 \mathrm{mM}$ EDTA, $1 \mathrm{mM}$ PMSF, and $1 \%$ octyl- $\beta$-D glucopyranoside in the presence of protease inhibitors in PBS at $4{ }^{\circ} \mathrm{C}$ for $2 \mathrm{~h}$. The lysate was precleared $\left(2000 \times g, 5 \mathrm{~min}\right.$ at $\left.4{ }^{\circ} \mathrm{C}\right)$ and cleared by centrifugation at $20,000 \times g, 40 \mathrm{~min}$ at $4{ }^{\circ} \mathrm{C}$ prior to loading to the immunoaffinity column (AminoLink, Pierce) with covalently linked antibody. Following binding, the affinity column was washed using 7 column volumes of each buffer (150 mM NaCl, $20 \mathrm{mM}$ Tris-HCl; $400 \mathrm{mM} \mathrm{NaCl}, 20 \mathrm{mM}$ Tris-HCl; $150 \mathrm{mM}$ $\mathrm{NaCl}, 20 \mathrm{mM}$ Tris- $\mathrm{HCl}$ and $20 \mathrm{mM}$ TrisHCl, pH 8.0), and bound complexes were eluted in $0.1 \mathrm{~N}$ acetic acid.

\subsection{Purification and Concentration of MHC-I Peptides}

Eluted peptide MHC-I complexes were desalted using Sep-Pak-C18 cartridges (Waters). Cartridges were prewashed with $80 \%$ acetonitrile in $0.1 \%$ trifluoro acetic acid (TFA) and with $0.1 \%$ TFA prior to loading of the sample and then with $0.1 \%$ TFA. The peptides were purified from MHC-I complex by elution with $40 \%$ acetonitrile in $0.1 \%$ TFA prior to drying the samples using vacuum centrifugation (Eppendorf, Hamburg, Germany).

\subsection{LC-MS Analysis of MHC-I Peptides}

Each dry sample was dissolved in $11 \mu \mathrm{L}$ of LCMS solvent A (97\% water, $3 \%$ acetonitrile, $0.1 \%$ formic acid), of which $10 \mu \mathrm{L}$ was injected into a C18 guard desalting column (Acclaim pepmap 100, $75 \mu \mathrm{m} \times 2 \mathrm{~cm}$, nanoViper, Thermo, Waltham, MA, USA). After 5 min of flow at $5 \mu \mathrm{L} / \mathrm{min}$ with the loading pump, the 10-port valve was switched to analysis mode, in which the NC pump provided a flow of $250 \mathrm{~nL} / \mathrm{min}$ through the guard column. The linear gradient then proceeded from $2 \%$ solvent B (95\% acetonitrile, $5 \%$ water, $0.1 \%$ formic acid) to $25 \%$ B in 90 min followed by wash at $99 \% B$ and re-equilibration. Total LC-MS run time was $123 \mathrm{~min}$. We used a nano EASY-Spray column (pepmap RSLC, C18, $2 \mu \mathrm{m}$ bead size, $100 \AA$, $75 \mu \mathrm{m}$ internal diameter, $50 \mathrm{~cm}$ long, Thermo) on the nano electrospray ionization (NSI) EASY-Spray source (Thermo) at $60^{\circ} \mathrm{C}$. One raw file (20190121) was generated in the same system but using a shorter column and shorter gradient, $15 \mathrm{~cm}$ and $30 \mathrm{~min}$, respectively. Online LC-MS was performed using a hybrid Orbitrap Fusion mass spectrometer (Thermo Scientific, Waltham, MA, USA). FTMS master scans in profile mode with 120,000 resolution (and mass range 300-750 m/z) were followed by data-dependent MS/MS in centroid mode on the top 10 ions using collision-induced dissociation (CID) at 32\% normalized collision energy, activation time of $10 \mathrm{~ms}$, and activation $\mathrm{Q}$ of 0.25 . Precursors were isolated with a $2 \mathrm{~m} / \mathrm{z}$ window. Automatic gain control (AGC) targets were 1e6 for MS1 and 1e4 for MS2. Maximum injection times were $100 \mathrm{~ms}$ for MS1 and $100 \mathrm{~ms}$ for MS2. Dynamic exclusion was used with $30 \mathrm{~s}$ duration. Only precursors with charge state 2-4 were selected for MS2.

\subsection{Proteomics Database Search}

MS/MS spectra were searched by Byonic v3.6.0 (Protein Metrics Inc., Cupertino, CA, USA), using a target-decoy strategy. For the first search for tumor-associated antigens, the database used was the Uniprot mouse reference protein database $(53,378$ protein 
entries, including Swissprot and some TrEMBL entries, downloaded from uniprot.org on 20180917). Precursor mass tolerance of $10 \mathrm{ppm}$ and product mass tolerance of $0.36 \mathrm{Da}$ for CID-ITMS2 were used. No enzyme specificity was used, and oxidation of methionine (common2) and phosphorylation on serine, threonine, or tyrosine (rare2) were used as variable modifications. Maximum precursor mass was 1500, with only 1 precursor per MS2 spectrum allowed, and a smoothing width of $0.01 \mathrm{~m} / \mathrm{z}$. False discovery rate (FDR) cutoff of $5 \%$ was employed at peptide level.

For the discovery of non-canonical peptides (ERV antigens), we performed re-search against the wider Uniprot database containing all Swissprot and all TrEMBL murine protein entries (restricted to taxonomy 10090, Mus musculus, containing a total of 92,607 sequences, downloaded from uniprot.org, accessed on 6 October 2020). The settings were the same as above, except that phosphorylations were not considered as modifications.

We validated the identification of selected endogenous peptides with synthetic peptides and produced mirror plots showing endogenous and respective synthetic peptide MS2 spectra. For each endogenous/synthetic MS2 spectrum pair, quality scores pertaining to the peptide-spectrum match are displayed (Precursor error, DeNovoScore, MSGFScore, SpecEvalue, Evalue). These were obtained by using an MSGF+ [13] search against a database containing only the peptides of interest.

The mass spectrometry proteomics data have been deposited to the ProteomeXchange Consortium via the PRIDE partner repository with the dataset identifier PXD016112.

\subsection{In Silico Analysis of the MHC-I Peptides}

Peptide motif analysis was performed using Gibbs clustering analysis [14] at the DTU bioinformatics server (tool version 2.0). Unsupervised alignment and clustering of input peptides ( 9 mers, 10 mers or 11 mers separately) as $1-5$ clusters and respective motif lengths was carried out using default settings. The known H-2Kd and H-Dd motifs were obtained from DTU Bioinformatics NetMHC 4.0 Motif Viewer [15]. MHC-I binding affinity predictions of 9 mers was performed using the IEDB resource tools ('IEDB recommended $2.19^{\prime}$ method). To identify MHC-I peptide-enriched biological processes, 9 mers were mapped back to their source proteins, and overrepresentation analysis was performed using PANTHER (version released 20190711) [16]. Mouse proteome was used as background (reference list Mus musculus, all genes in database) and 'GO biological process complete', 'GO molecular function complete', and 'GO cellular component complete' (release 3 July 2019) as the annotation data set using Fisher's exact test with FDR correction.

\subsection{Mice and Animal Experiment}

The animal experiment was approved by the Experimental Animal Committee of Southern Finland National Animal Experiment Board, ELLA, (license number ESAVI/9817/ 04.10.07/2016) and was performed under the guidelines of the of the Regional State Administrative Agencies in Finland. Age-matched 4-6 weeks old female Balb/c OlaHsd mice (Envigo, Venray, The Netherlands) were used as the syngeneic mouse tumor model of 4T1 TNBC. Mice were immunized three times $(15,12$, and 4 days prior to tumor engraftment) by s.c injection of peptide pools plus adjuvant (5-6 peptides per pool, á $25 \mu \mathrm{g}$ peptide plus $100-125 \mu \mathrm{g}$ poly I:C (Vaccigrade, InvivoGen, Toulouse, France)). The used peptide pools are as depicted in Table 1. Peptides were purchased as custom synthesis from Zhejiang Ontores Biotechnologies Co. (Hangzhou, Zhejiang, China). To engraft the tumors, $3 \times 10^{5} 4 \mathrm{~T} 1$ cells in PBS were injected s.c. in the right flank of the animals. Tumor growth was measured every second day using a digital caliper once the tumors became palpable, and tumor volume was calculated using the formula: (length $\times$ width $\left.{ }^{2}\right) / 2$. Tumors were allowed to grow for 21 days until the mice were sacrificed, and the tumors and spleens were collected for further analysis. Percent tumor growth inhibition (\%TGI) was defined as the difference between the median tumor volume (MTV) of the adjuvant and immunization groups, using the formula: \%TGI $=(($ MTVadjuvant-MTVimmunised $/$ MTVadjuvant $)) \times 100$. Additionally, individual mice were scored as responders (at least 30\% decrease in tumor volume in 
comparison to the median tumor volume of the adjuvant group) or non-responder (at least $20 \%$ increase in tumor volume in comparison to the median tumor volume of the adjuvant group) at the study endpoint.

Table 1. Peptide characteristics and peptide pools used for therapeutic vaccination.

\begin{tabular}{|c|c|c|c|c|c|c|c|}
\hline$\#$ & Peptide & UniProt ID & Protein Description & $\begin{array}{l}\text { Short } \\
\text { Name }\end{array}$ & $\begin{array}{c}\text { H-2Kd IC50 } \\
\text { (nM) }\end{array}$ & $\begin{array}{l}\text { Known MHC-I } \\
\text { Lgand (IEDB) * }\end{array}$ & $\begin{array}{l}\text { MHC-I Ligand } \\
\text { in Normal } \\
\text { Tissue }\end{array}$ \\
\hline \multicolumn{8}{|c|}{ Peptide pool1 } \\
\hline$\# 1$ & RYLPAPTAL & Q9JL70 & Fanconi anemia group A protein homolog & Fanca & 13.44 & Yes & - \\
\hline \#2 & FYITSRTQF & F8WI90 & Tyrosine-protein kinase & Scr & 15.05 & Yes & Yes \\
\hline \#3 & SYFPEITHI & B1ASP2 & Tyrosine-protein kinase & Jak1 & 21.79 & Yes & - \\
\hline$\# 4$ & FYLETQQQI & Q99MP8 & BRCA1-associated protein & Brap & 29.37 & - & - \\
\hline \#5 & NYVPGKFTV & E9PXX8 & Metastasis-associated in colon cancer 1 & Macc1 & 59.45 & - & - \\
\hline \multicolumn{8}{|c|}{ Peptide pool2 } \\
\hline \#6 & EYVHTKNFI & H7BXB1 & Casein kinase I isoform alpha & Csnk1a1 & 65.81 & - & - \\
\hline \#7 & NYQDTIGRL & A0A0A6YWC8 & Vimentin & Vim & 470.08 & Yes & Yes \\
\hline \#8 & KYLATLETL & B1ASP2 & Tyrosine-protein kinase & Jak1 & 13.72 & Yes & Yes \\
\hline \#9 & YFISSTTRI & A0A0A0MQ80 & Spermatogenesis-associated protein 5 & Spata5 & 29.64 & Yes & - \\
\hline \#10 & SYLKSELGL & A2AQD5 & Sperm-specific antigen 2 homolog & Ssfa2 & 121.09 & - & - \\
\hline \multicolumn{8}{|c|}{ Peptide pool3 } \\
\hline \#11 & SYHPALNAI & S4R1L5 & Baculoviral IAP repeat-containing protein 6 & Birc6 & 9.9 & Yes & - \\
\hline \#12 & SYYAVAHAV & A0A0R4J170 & Transcription activator BRG1 & Smarca4 & 11.16 & - & - \\
\hline \#13 & AYKAVLNYL & D3YXN3 & Testis-expressed protein 30 & Tex30 & 43.77 & Yes & - \\
\hline \#14 & EYVANLTEL & A0A0R4J170 & Transcription activator BRG1 & Smarca4 & 100.38 & Yes & - \\
\hline$\# 15$ & KYSAQIEDL & B1AUF1 & Ski oncogene & Ski & 443.63 & - & - \\
\hline \multicolumn{8}{|c|}{ Peptide pool4 } \\
\hline \#16 & EYIHSKNFI & Q9JMK2 & Casein kinase I isoform epsilon & Csnk1e & 26.77 & Yes & - \\
\hline$\# 17$ & KYQAVTATL & P19253 & $60 S$ ribosomal protein L13a & Rpl13a & 14.51 & Yes & Yes \\
\hline \#18 & KYQEALDVI & Q8BWZ3 & $\begin{array}{c}\text { N-alpha-acetyltransferase } 25, \text { NatB auxiliary } \\
\text { subunit UV excision repair protein RAD23 } \\
\text { homolog B, HR23B }\end{array}$ & Naa25 & 31.04 & Yes & - \\
\hline \#19 & SYENMVTEI & P54728 & $\mathrm{mHR} 23 \mathrm{~B}$ & $\operatorname{Rad} 23 b$ & 9.65 & Yes & - \\
\hline \#20 & SYKPIVEYI & Q8C1B7 & Septin 11 & Sept11 & 45.35 & Ye & - \\
\hline \#21 & TYVPIAQQV & A2APB8 & Targeting protein for $\mathrm{Xklp} 2$ & Tpx2 & 90.44 & Yes & - \\
\hline
\end{tabular}

* IEDB (Immune Epitope Database), ** Described as HLA-I ligand in the 'HLA Ligand Atlas'.

For the therapeutic experiment, the PeptiCRAd vaccine platform combining an oncolytic adenovirus and polyK peptide epitope was used in the context of established 4T1 tumors. VALO-mD901 adenovirus ([17] Ad5/3, delta 24, delta E3-CR1-alpha, gp19K and 14.7 K genes) expressing murine Cd40L and Ox40L under CMV-promoter was utilized as the PeptiCRAd platform. PeptiCRAd formulation was prepared as previously described by complexing the oncolytic adenovirus with polyK peptides for $15 \mathrm{~min}$ at room temperature [18] and used within 30 min following complex formation. For TAA-PeptiCRAd, VALO-mD901 was complexed with KKKKKKKFYLETQQQI, KKKKKKSYHPALNAI, and KKKKKKYQAVTATL peptides and for ERV-PeptiCRAd with KKKKKFYLPTIRAV and KKKKKKKTYVAGDTQV peptides. These peptides respond to the initially discovered peptides FYLETQQQI, SYHPALNAI, and KYQAVTATL originating from Brap, Birc6, and Rpl13a source proteins, respectively (experiment shown in Section 3.4) and peptides FYLPTIRAV and TYVAGDTQV originating from the ERV source genes (experiment shown in Section 3.5). Viral dose was $1 \times 10^{9} \mathrm{vp}$ complexed with $8 \mu \mathrm{g}$ peptide (total peptide amount) per tumor. PeptiCRAd was administered intratumorally, anti-PD-1 intraperitoneally, and PBS was used as mock injections. PolyK-tailed peptides were purchased as custom synthesis from Ontores Zhejiang Ontores Biotechnologies Co. (Hangzhou, Zhejiang, China) (tumor-associated antigens) and GeneScript (endogenous retrovirus antigens). 


\subsection{Immunogenicity Analysis}

Splenocytes from tumor-bearing animals were harvested at the study endpoint from the therapeutic experiment. Splenocytes were smashed and processed into single cells through a 70 micron cell strainer. Immune cell reactivity towards individual peptides was tested as IFN-gamma secretion upon re-stimulation using ELISPOT (ImmunoSpot, CTL Europe GmbH, Bonn, Germany). A total of $1 \times 10^{6}$ splenocytes were re-stimulated for 2 days with $1 \mathrm{ng}$ individual peptides. ELISPOT plate manufacturer instructions were used for staining of the plate, and the spots were read and quantified by ImmunoSpot (CTL Europe GmbH, Bonn, Germany).

\subsection{Flow Cytometric Analysis of Tumor-Infiltrating Lymphocytes}

The tumors were digested with collagenase and DNAse $\left(1 \mathrm{~h},+37^{\circ} \mathrm{C}\right)$ and pushed through a 70 micron cell strainer. Non-specific binding of immunoglobulin to the $\mathrm{FC}$ receptors was blocked with anti-mouse CD16/32 (TruStain Fc block, Biolegend, CA, USA). The tumors were stained using antibodies against: CD3 (clone 145-2C11), CD8 (clone 53-6.7), PD-1 (clone 29F.1A12), and Tim3 (clone B8.2C12) (Biolegend) at $+4{ }^{\circ} \mathrm{C}$ for $45 \mathrm{~min}$. Flow cytometric acquisition of $1 \times 10^{6}$ events was performed using BD LSRFortessa (BD Biosciences) and was analyzed using FlowJo 10.4 software (Ashland, Willington, DE, USA).

\subsection{Statistical Analysis}

Statistical analysis was performed using GraphPad Prism 6.0 software (GraphPad version 8.2.0 Software, San Diego, CA, USA). For tumor growth curve analysis, 2-way ANOVA with Tukey's multiple comparison was used, and $p$-values $<0.05$ were considered as statistically significant. For flow cytometric data analysis, an unpaired $t$ test was used. Results are expressed as mean of the group \pm standard error mean (SEM).

\section{Results}

\subsection{Direct Identification of Tumor-Associated Antigens in Mouse Triple-Negative Breast Tumor}

Therapeutic cancer vaccination using neoantigens has demonstrated clinical benefit in highly immunogenic tumors with high levels of somatic mutations such as melanoma. In tumor types with low mutational burden, the likelihood of identifying neoepitopes amenable for therapeutic targeting is more limited. This limitation calls for identifying novel, effective, shared tumor-associated antigens for tumor types susceptible for immunotherapy in order to expand the current immunomodulatory strategies to benefit wider patient groups. In this regard, TNBC is an attractive model with shown sensitivity to checkpoint blockade.

To identify naturally presented tumor-associated antigens for a TNBC model, we performed immunopeptidome analysis on MHC class I (MHC-I) bound peptides using the murine 4T1 cell line (Figure S1A). MHC-I peptide complexes were immunoaffinity purified, and eluted peptides were analyzed by tandem mass spectrometry. Using the murine reference proteome (uniprot.org 53,378 entries) as the search database, we found a total of 2207 MHC-I-associated peptide sequences using an FDR threshold of $5 \%$ for peptide identification. The depth of our immunopeptidome analysis is in line with recent publications identifying $\mathrm{H}-2 \mathrm{~K}^{\mathrm{b}}$ and $\mathrm{H}-2 \mathrm{D}^{\mathrm{b}}$ restricted immunopeptidomes for several murine tumor cell lines and mice tissues $[19,20]$. Next, we performed bioinformatics analysis of the identified MHC-I peptides and their source proteins (Figure S1B). The peptides represent the typical length distribution of isolated MHC-I ligandomes (Figure 1b) with 9 mers as the most enriched peptide species. Of the unique 9 mers, $75 \%$ are known $\mathrm{H}-2 \mathrm{Kd}$ or $\mathrm{H}-2 \mathrm{Dd}$ ligands (described as Mus musculus epitopes in IEDB database 20210512). Gibbs clustering of the 9 mers, 10 mers, and 11 mers showed the peptides clustered into two distinct groups with preference for reduced amino acid complexity for residues at positions $\mathrm{P} 2$ and $\Omega$ or P2, $\mathrm{P} 3$, and $\Omega$ (Figure $1 \mathrm{c}$ and Figure S1C). These data are in line with known $\mathrm{H}-2 \mathrm{~K}^{\mathrm{d}}$ and $\mathrm{H}-2 \mathrm{D}^{\mathrm{d}}$ binding motifs with respect to their anchor residue positions and amino acid characteristics. Due to the high sensitivity of the mass spectrometry, we identified also longer peptides 
(12 mers-14 mers), which may additionally represent contaminating co-purifying peptides among a few 'true' MHC-I ligands.

We next aimed to identify the high-confidence MHC-I ligands among the 9 mers. Prediction of the peptide binding affinity to MHC-I showed $67 \%$ of the 9 mers bound either H-2 Kd or H-2 Dd and can thus be considered as MHC-I ligands (using the $<500$ nM IC50 affinity value as cutoff for MHC-I binding, Figure 1d). The vast majority of these ligands showed clear preference to $\mathrm{H}-2 \mathrm{Kd}$, and $41 \%$ of the $\mathrm{H}-2 \mathrm{Kd}$ ligands bound $\mathrm{H}-2 \mathrm{Kd}$ with high affinity (<50 nM IC50, Supplementary Data File S1).

The MHC-I-presented peptides are derived from the degradation of cellular proteins, and as such the immunopeptidome reflects the cellular events or cell status. During malignant transformation, various cellular processes become deregulated [21,22] leading to abnormal protein production. The presented peptide ligands derived from deregulated proteins may offer an attractive target for immunotherapy, as they may present tumorselective epitopes altered, e.g., in abundance in comparison to normal cells. In our 4T1 immunopeptidome, the identified 9 mer ligands are derived from various different source proteins with the majority of source proteins producing only one detectable MHC-I ligand. We observed few exceptions, which showed a wider presentation coverage (maximum of 4 assigned MHC-I ligands). The source proteins are enriched in nuclear and intracellular proteins with various enzymatic or nucleotide binding activities. The source proteins have a function in various biological processes, mainly reflecting the high proliferative, metabolic, and biosynthetic status of the cells (Figure 1e).

\subsection{Discovery of MHC-I-Presented Endogenous Retroviral Antigen}

A particularly interesting class of tumor antigens may arise from aberrant translation in tumor tissue. These tumor-selective protein products are generated from genomic loci previously considered as non-coding, intronic, or are products of un-annotated alternative gene translations. Remarkably, the presence of aberrant translation products has recently been demonstrated in various tumor types, including human breast tumors [23]. Importantly, these candidate tumor antigens can generate specific T-cell responses towards the tumor with clinical relevance $[11,24]$.

We thus re-searched our mass spectrometry ligandome data files against a database containing the entire unreviewed TrEMBL set from Uniprot, a total of 92,607 protein entries (all Mus musculus Swissprot + TrEMBL entries) (Figure S1A). Many of the TrEMBL entries that do not map to canonical genes are potentially the product of aberrant transcription and translation in tumors. Applying an FDR cutoff of 5\%, as before, resulted in $2481 \mathrm{MHC}$ peptides found (Supplementary Data File S1). These peptides showed typical MHC-I ligand characteristics in terms of peptide length, amino acid enrichment, and predicted binding efficiency to MHC-I. However, this allowed the discovery of additional MHC peptides mapping to TrEMBL entries (including peptides not found in the reference proteome searches), most of which actually correspond to canonical proteins, but one belonged to theoretically non-coding genomic regions. This peptide, FYLPTIRAV (confidence score $\mid \log$ prob $\mid=4.75$ ) from hypothetical viral gag protein Q811J2 (Uniprot accession), maps to an endogenous retroviral (ERV) region, LOC72520. We further validated the identification of FYLPTIRAV peptide with a synthetic one, and the mirror plots showing endogenous peptide and respective synthetic peptide are presented as Supplementary Data File S2.

\subsection{Control of Tumor Growth by MHC-I Ligand Immunization}

Tumor regression can be induced by therapeutic cancer vaccination targeting tumorselective epitopes, and in experimental tumor models marked responses are achievable even when using a single immunodominant epitope. In case of a highly heterogeneous tumor, such as TNBC, the identification of immunodominant epitopes able to target the majority of the tumorigenic cells may represent a challenge. Thus, tumor vaccines can be designed as multivalent to obtain protective T-cell responses against a larger repertoire of tumor antigens for eliciting sustainable immunotherapy responses. 
We studied the antitumorigenic potential of peptide-based vaccination utilizing the identified tumor associated antigens in BALB/c mice. The tumor antigens for the vaccine formulations were selected according to predicted high affinity of peptide binding to MHC-I. Additionally, peptides originating from source proteins having a function in cancer biology (such as Ski) were included despite showing lower putative MHC-I binding (Table 1). Additionally, one group (peptide pool \#4) was selected based on an in-house developed tool to estimate peptide immunogenicity using peptide similarity to viral epitopes [25]. In short, peptide similarity to viral peptides was computed via pairwise weighted alignment. Peptide central section similarity was prioritized, as being mostly involved in peptide/TCR interaction. Moreover, peptide putative binding affinity to MHCI was included within the immunogenicity estimate. Hence, the peptide pool 4 peptides were chosen based on similarity to viral peptides and high putative binding to MHC-I as surrogate to peptide immunogenicity.

Mice were immunized thrice with peptide pools (5-6 MHC-I restricted peptides per pool, Table 1) using poly I:C as an adjuvant. An irrelevant peptide plus adjuvant was used as mock control.

Following immunization, mice were challenged with 4T1 tumor cells, and tumor growth was measured (Figure 2a). We observed reduced tumor growth in two immunization groups (Peptide pool 3 and Peptide pool 4) with 44\% and 36\% tumor growth inhibition at the endpoint in comparison to the adjuvant control (Figure 2b). The effect in tumor growth was statistically significant in these immunization groups, with $62.5 \%$ and $50 \%$ of individual mice showing antitumorigenic responses, respectively (Figure 2c and Figure S2). In Peptide pool 1 two mice developed small tumors following immunization. However, as a group, the effect on tumor control was not markedly different from the adjuvant controls. Of note, one peptide immunization group (Peptide pool2) showed larger tumors than the adjuvant group. This undesirable effect emphasizes the need to acquire sufficient knowledge on the antigens and their impact on the tumor and immune biology.

The moderate antitumor responses prompted us to study the correlation of the individual responses with frequency and phenotype of tumor-infiltrating immune cells. FACS analysis on tumor infiltrating lymphocyte (TIL) relative frequency showed comparable CD8+ T-cell infiltration among the different treatment groups (on average $0.19-0.34 \%$ of the tumor cells stained CD8-positive) (Figure 3a). Peptide vaccination did not increase CD8+ T-cell infiltration in comparison to the adjuvant group, and no clear correlation between individual tumor size and CD8+ cell infiltration was observed (Figure S3A). Thus, even though immunization using tumor antigens elicited antitumor control, this was not associated with strong overall increase in immune cell infiltration within the tumors.

Tumor-infiltrating immune cells express multiple co-stimulatory and inhibitory receptors in response to T-cell receptor (TCR) stimulation. Antigen exposure triggers the initial clonal expansion of antigen-specific T-cell populations. However, under persistent exposure to the antigen co-expression of several inhibitory receptors, T-cells are rendered dysfunctional. This T-cell 'exhaustion' is observed during chronic viral infection [26,27] as well as in response to tumor antigen exposure, either spontaneous or tumor vaccineinduced $[28,29]$. Concomitantly, expression of the inhibitory receptors can be used as a surrogate marker for detecting and identifying the tumor reactive repertoire of CD8+ cells from the bulk TILs $[30,31]$. Especially interesting in this respect is the co-expression of PD-1, LAG-3, and TIM-3 on CD8+ TILs, as these are shown to phenotypically identify tumorreactive CD8+ lymphocytes, regardless of antigen specificity [30]. We initially analyzed the PD-1-expressing CD8+ cells among the TILs. The vast majority of CD8+ cells expressed PD-1 with no clear increase in the frequency of PD-1+ cells in the vaccination groups versus control (Figure S3B). However, the CD8+ cells in the peptide vaccinated mice expressed PD-1 at significantly higher levels in comparison to the adjuvant controls (Figure 3b), suggesting a qualitative difference in these tumor infiltrating cells. A subpopulation of the PD-1+ CD8+ cells additionally co-expressed TIM-3, defining exhausted CD8+ T-cells (Figure 3c). Interestingly, we observed higher prevalence of the PD-1+ Tim-3+ double- 
positive CD8+ cells associated with smaller tumor size, suggesting this subpopulation of immune cells may be important for tumor control activity (Figure 3d).

a

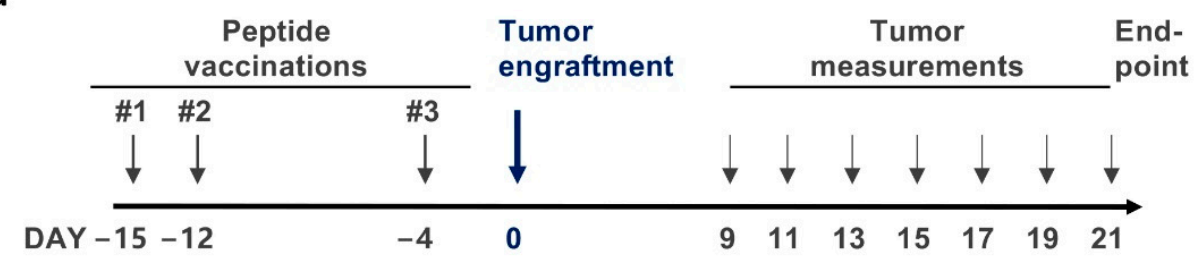

b

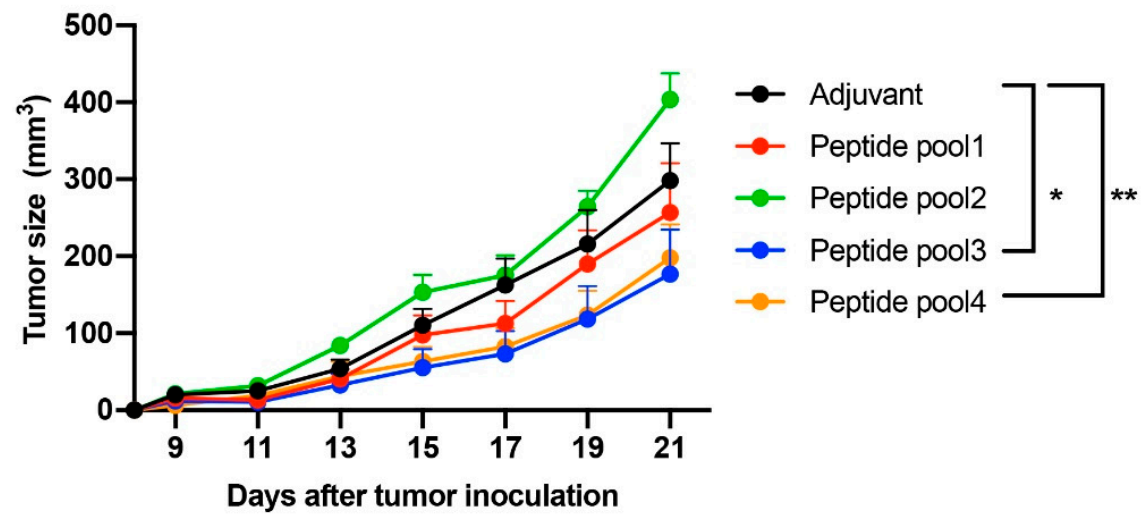

C

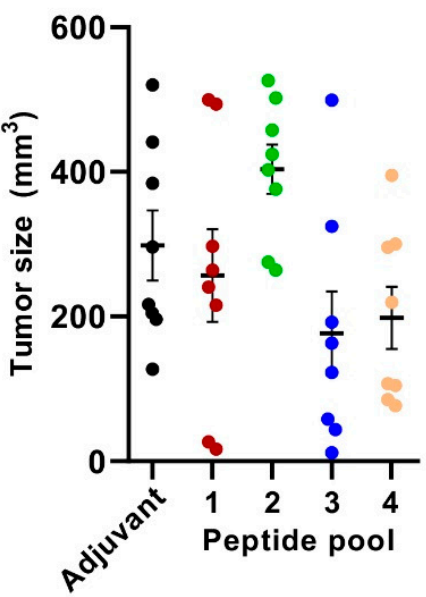

Figure 2. Tumor control potential of tumor-associated antigens. (a) Animal experiment treatment schedule. Balb/c mice were immunized with an adjuvant (poly I:C) in combination with irrelevant peptide (SIINFEKL that binds to $\mathrm{H}-2 \mathrm{~Kb}$ and not the BALB/c expressed $\mathrm{H}-2 \mathrm{Kd}$ or $\mathrm{H}-2 \mathrm{Dd}$ (Adjuvant group) or a pool of 5-6 peptides (groups: Peptide 1, 2, 3 and 4; 25 ug each peptide per pool)). Following three peptide vaccinations, 4T1 tumors were engrafted (300,000 cells/tumor). Tumor measurements were initiated once the tumors became palpable. (b) Tumor growth curves for immunization groups. Peptide pool 3 and Peptide pool 4 group vaccinations control tumor growth in the aggressive 4T1 model, as compared to mock immunization. Statistical analysis of tumor growth curves was performed using two-way Anova, ${ }^{*} p<0.05,{ }^{* *} p<0.01$. The error bars show SEM, $n=8$. (c) Individual tumor sizes at the endpoint (day 21 after tumor engraftment) are presented. 

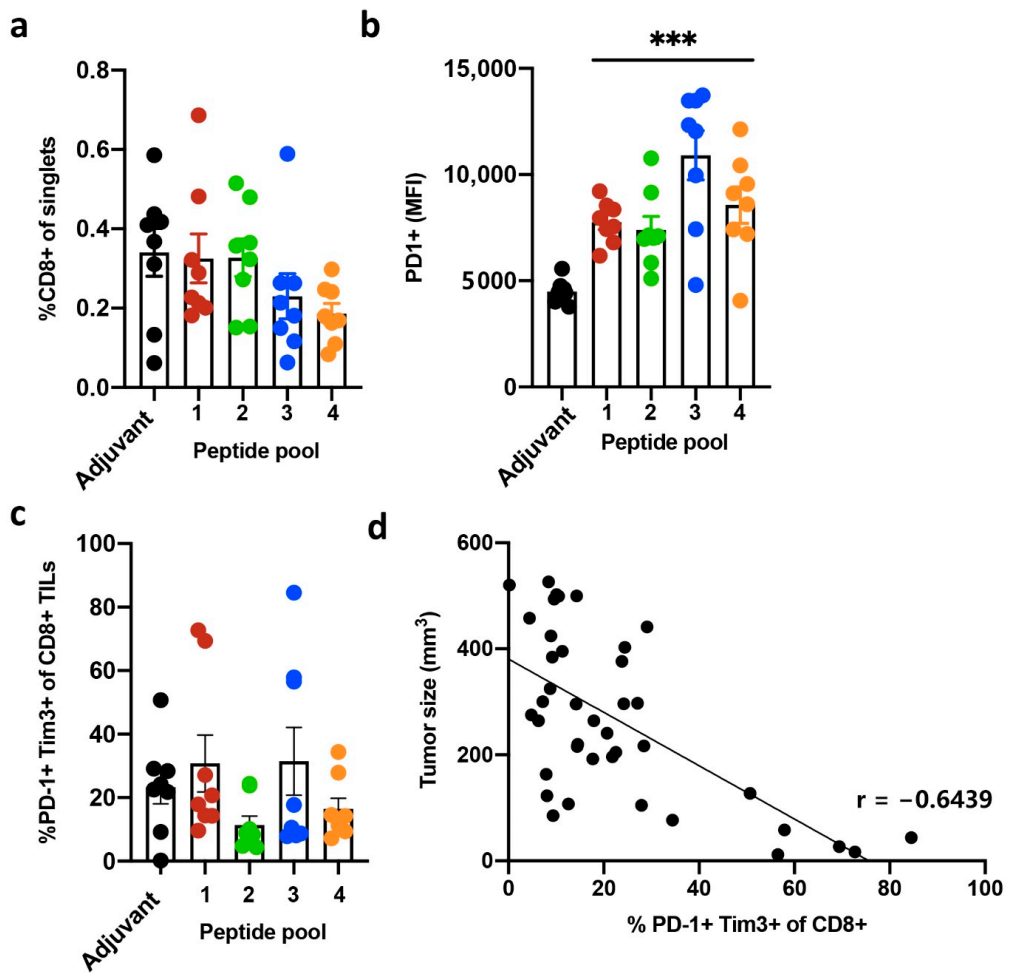

Figure 3. Flow cytometric analysis of tumor-infiltrating CD8+ T-cells. Dots represents analysis of individual tumors, and bars represent the mean of the group. (a) Percent of CD8+ cells of the singlets. (b) Median expression of PD-1 in CD8+ T-cells. Statistical analysis was performed using unpaired, two-tailed $t$-test, ${ }^{* * *} p<0.006$. (c) Percent PD-1+ and Tim3+ double-positive CD8+ cells. (d) Correlation analysis of tumor size and percentage of PD-1+ Tim3+ CD8+ cells. The tumor size (day 21) negatively correlates with percentage of infiltrated PD-1+ Tim3+ double positive CD8+ cells. Pearson correlation, $\mathrm{r}=-0.6439$.

\subsection{Therapeutic Vaccination with PeptiCRAd Cancer Vaccine Targeted to Tumor-Associated Antigens Controls Tumor Growth}

We reasoned that the immunosuppressive nature of the tumor model may require direct modulation of the tumor microenvironment, as achieved with oncolytic viruses or checkpoint inhibition, for enhanced T-cell mediated immunity to control tumors [32,33]. We have previously shown increased presence of antigen-specific TILs within tumors with less exhausted phenotype following immunization with a novel cancer vaccine platform PeptiCRAd [17,33]. PeptiCRAd technology combines an oncolytic adenovirus and tumor antigen for enhanced tumor-specific CD8+ T-cell responses towards tumors [18]. Tumor antigen targeting is achieved by adsorbing the tumor peptide onto the oncolytic viral capsid to guide the specificity of the vaccine-induced immune response. Our preliminary results support generation of improved antigen-specific responses with the PeptiCRAd platform over peptide-Poly I:C vaccination (Figure S4).

To further investigate the therapeutic potential of tumor-associated antigen cancer vaccination as immunotherapy, we applied the PeptiCRAd technology. Additionally, we tested the platform in a therapeutically more challenging setting with pre-established tumors. Tumor-bearing mice were vaccinated intratumorally with PeptiCRAd complexed with the identified tumor-associated antigens (TAA-PeptiCRAd group) or with the oncolytic virus (Virus group) alone or in combination with checkpoint inhibition (Virus + a-PD-1 and TAA-PeptiCRAd + a-PD-1) (Figure 4a). The immunopeptidomics discovered peptides FYLETQQQI, SYHPALNAI, and KYQAVTATL originating from Brap, Birc6, and Rpl13a source proteins, respectively, were used as antigens for the TAA-PeptiCRAd. This therapeutic vaccination led to significant protection in TAA-PeptiCRAd-receiving mice over the virus control (Figure $4 b, c, p<0.01$ ) with $90 \%$ of mice showing potent antitumor activity (Figure S5). 
The combination of PeptiCRAd with checkpoint inhibition (anti-PD-1 antibody) did not markedly increase the overall therapy response over PeptiCRAd treatment despite conferring the highest systemic T-cell responses against the tumor-associated antigens (Figure 4d).

a
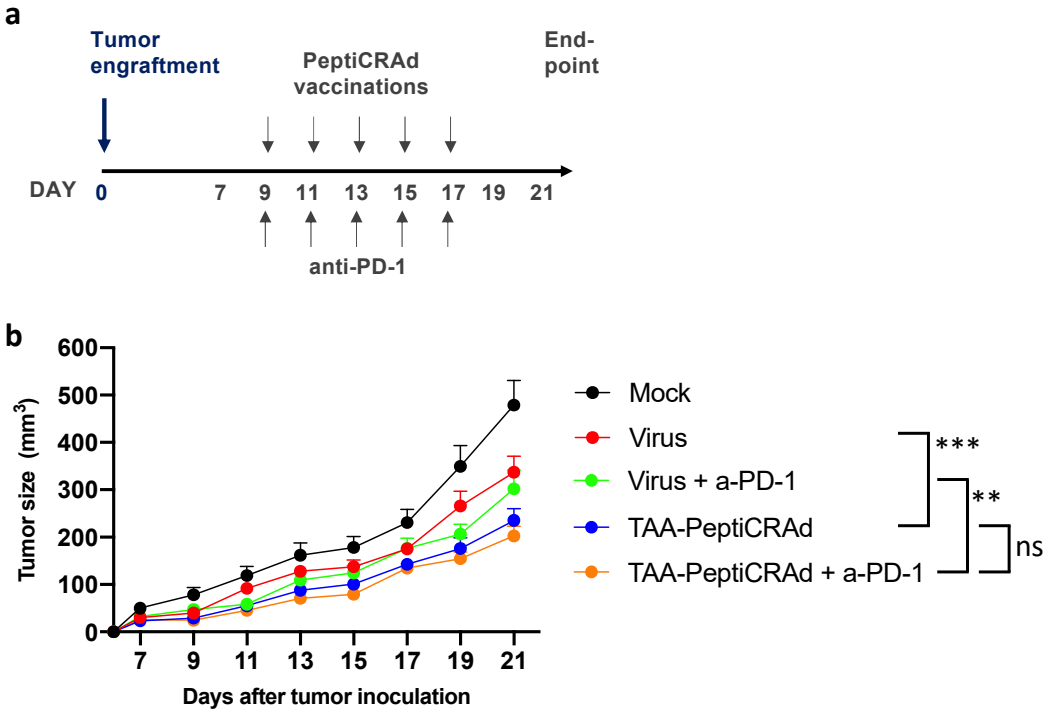

c

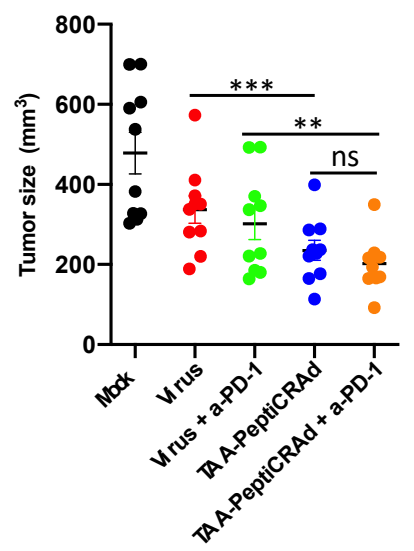

d

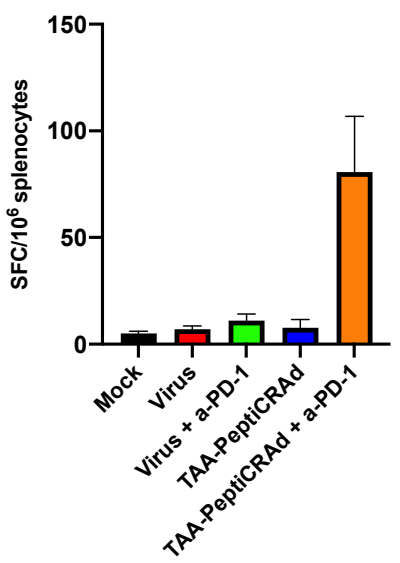

Figure 4. Therapeutic vaccination with PeptiCRAd targeted to tumor-associated antigen induces anti-tumor activity in 4T1 model. (a) Animal experiment treatment schedule. Balb/c mice were engrafted with $4 \mathrm{~T} 1$ tumors (300,000 cells/tumor). Mice were assigned to treatment groups at day 7 blindly. Treatments were initiated at day 9 once the tumors were palpable. Each mouse received 5 injections of virus or PeptiCRAd (i.t.) and 5 injections of anti-PD-1 (i.p.) or PBS (mock injections). (b) Tumor growth curves for treatment groups. Treatment groups: mock injected (Mock); PeptiCRAd virus backbone without peptide loading (Virus); PeptiCRAd loaded with tumor-associated antigens (TAA-PeptiCRAd) with or without anti-PD-1 (a-PD-1). The immunopeptidomics discovered peptides FYLETQQQI, SYHPALNAI, and KYQAVTATL originating from Brap, Birc6, and Rpl13a source proteins, respectively, were used as antigens for the TAA-PeptiCRAd, and poly-lysine-tail was added to the endogenous peptides to allow PeptiCRAd complex to formulate. Statistical analysis of tumor growth curves was performed using two-way Anova with Tukey's multiple comparison, ${ }^{* *} p<0.05$, *** $p<0.01$. The error bars show SEM, $n=10$. (c) Individual tumor sizes at the endpoint (day 21 after tumor engraftment) are presented. (d) Immune responses against tumor-associated antigens at endpoint. Splenocytes were harvested at the end of the therapeutic experiment from 3 mice from each treatment group and stimulated with the tumor-associated antigens peptides for 2 days. Inductions of peptide specific T-cell responses were analyzed by interferon-gamma enzyme-linked immunospot (ELISPOT). Spot forming colonies (SFC) were read and quantified at ImmunoSpot (CTL Europe). 
3.5. Therapeutic Vaccination with PeptiCRAd Cancer Vaccine Targeted to Endogenous Retroviral Antigen Shows Antitumor Efficacy

Encouraged by the marked antitumor protection in this tumor model, which is typically difficult to treat, we wished to explore further the $4 \mathrm{~T} 1$ tumor antigenic landscape for improved therapeutic efficacy using the identified ERV (Section 3.2). The ERV antigen is a potential example of a genomic region silenced in normal tissues but transcriptionally and translationally awakened in cancer cells. For instance, according to the Aceview NCBI resource of curated cDNA sequences, LOC72520 cDNA has been found in spontaneous mouse mammary tumors that metastasized to the lung [34]. Moreover, these types of normally non-coding genomic regions could potentially be recurring in many tumors, and as such may actually be shared antigens with high immunogenic potential. We therefore explored the therapeutic potential of the most confident example found here, the FYLPTIRAV peptide from ERV LOC72520 and the TYVAGDTQV peptide.

To this end, we designed a similar therapeutic experiment (similar treatment schedule as in Figure 4a) to study the therapeutic potential of these peptides in a therapeutic vaccination setting. PeptiCRAd was used as the vaccine platform and was complexed with the FYLPTIRAV and TYVAGDTQV peptides separately (ERV-PeptiCRAd). Mice receiving ERV-PeptiCRAd showed the highest tumor protection, which was statistically significant in comparison to the virus control $(p<0.001)$ (Figure 5a). Combination treatment with checkpoint inhibitor did not increase the level of protection, recapitulating our finding using the TAA-PeptiCRAd. The identified antigen induced weak, systemic immunological responses, as re-stimulation of splenocytes with these peptides showed measurable IFNgamma production in vaccinated mice (Figure $5 b$ ).

a

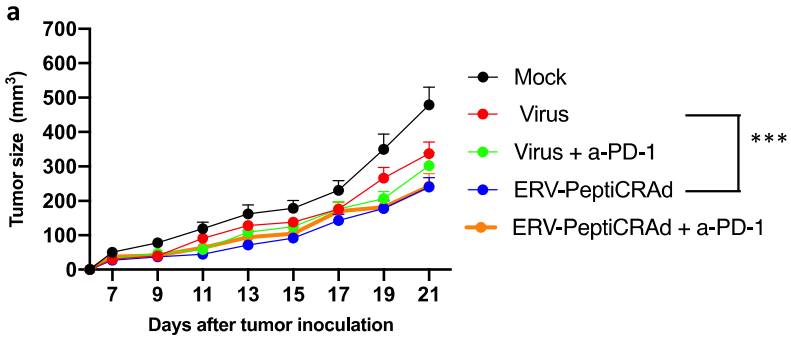

b

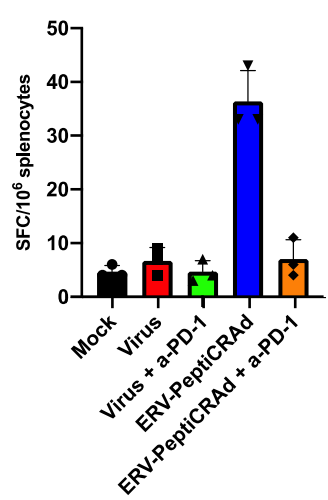

Figure 5. Therapeutic vaccination with PeptiCRAd targeted to endogenous retrovirus induces anti-tumor activity in the 4T1 model. (a) The animal experiment treatment schedule and tumor engraftment were the same as in Figure 4 experiment. Treatment groups: mock injected (Mock); PeptiCRAd virus backbone without peptide loading (Virus); PeptiCRAd loaded with endogenous retrovirus antigens (ERV-PeptiCRAd) with or without anti-PD-1 (a-PD-1). Statistical analysis of tumor growth curves was performed using two-way Anova with Tukey's multiple comparison, *** $p<0.01$. The error bars show SEM, $n=10$. (b) Immune responses against antigens at endpoint were analyzed as in Figure 4 using ELISPOT. Spot forming colonies (SFC) were read and quantified at ImmunoSpot (CTL Europe). 


\section{Discussion}

Recent technological advancements in mass spectrometry have made possible the direct immunopeptidome profiling and the identification of naturally presented T-cell epitopes from tumor material with high confidence. Here we identify tumor antigens using state-of-the-art mass spectrometry and further explore their potential as cancer vaccines in a prophylactic and therapeutic setting. The depth and quality of our immunopeptidome analysis are comparable to published immunopeptidome datasets on self-antigens in tumor cell lines and normal tissues sharing similar MHC-I restriction $[19,20]$. Of note, three of the peptides we described here were identical to HLA-I ligands recently discovered in an immunopeptidome study on human TNBC [35]. We observed that the majority of the MHC-I ligands' source proteins produced a single MHC-I ligand, with very few exceptions showing a wide presentation coverage. Thus, our study is in line with recent immunopeptidome analyses and offers a quality addition to the known MHC-I ligand data sets.

The advantage of utilizing direct identification for tumor antigen discovery is that the approach unveils the most clinically relevant target candidates for vaccine design. Recent successful clinical trials have validated this approach to produce candidate targets amenable as cancer vaccines with clinical benefit $[8,36]$. The obvious benefit of using tumorassociated antigens is that they can be 'shared' among patients. Of note, the utilization of 'shared' tumor-associated antigens does not restrict the personalization of immunotherapies, as the choice of antigens may be based on the characteristics of the patient's individual tumor (e.g., expression of specific tumor antigens or surrogate biomarkers). Moreover, additional cancer vaccination strategies based on neoepitopes from individual patients can be designed to later complement the personalized treatment. Candidate libraries of 'shared' antigens have recently been created for tumor types with low neoantigen load and successfully used as 'off-the-shelf' vaccines in clinical trials including glioblastoma [8] and TNBC [37]. However, care should be taken when choosing the antigen target not to induce any severe on-target off-tumor effects, leading to development of autoimmune reactions.

An exciting addition to the tumor antigen landscape is tumor-specific antigens derived from normally non-coding regions. Like tumor-associated antigens, these antigens could be shared between patients, be highly immunogenic, and present clinical targets for cancer T-cell recognition $[11,38]$. Our results warrant further studies on the prevalence, abundance, and most importantly MHC-I restricted presentation of antigens derived from annotated non-coding regions in human tumors and highlight the usefulness of accurate mass spectrometry-based approaches for their discovery.

We show here that cancer vaccines targeting multiple tumor antigens control tumor growth in aggressive mouse model of TNBC. Importantly, we show here for the first time that control of established tumors is achievable by integrating immunopeptidomic-based antigen discovery with the PeptiCRAd oncolytic adenovirus platform. This establishes a pipeline for improved oncolytic cancer immunotherapy by potentiating further personalization of oncolytic vaccine responses. The qualitative differences in the infiltrated T-cells as well presence of antigen-specific T-cell response in the therapeutic experiment suggest the vaccination protocols succeeded in triggering antitumor immunity. Modulation of the tumor microenvironment towards a more inflammatory state with the use of an oncolytic virus-based vaccine platform might have further sensitized TNBC cells to immune therapy $[32,33]$. However, due to the relatively modest systemic antigen-specific responses in vaccinated mice, we additionally evaluated spontaneous immune responses against the dominant CD8+ epitope in BALB/c models (gp70/AH1) [39] in the therapeutic vaccination. Response against gp70 was detected in PeptiCRAd-vaccinated mice, but not in mock-treated mice. Thus, despite the association of the vaccine-induced antigen-specific responses with anti-tumor activity, we cannot rule out the possibility of spontaneous immune responses playing an additional role in tumor control. Additionally, the anti-tumor effect may yet be improved, as the biological effects in the current study were modest despite obtaining statistical differences between the control and treatment groups. We 
anticipate further combination treatment, counteracting more efficiently the suppressive tumor microenvironment, may be required to further maintain the vaccine effect. Tim- 3 blockade would be especially intriguing in the context of triple-negative breast cancer as it additionally dampens the myeloid-derived suppressors [40]; however, exploring this in our model was out of the scope of the current study.

\section{Conclusions}

Our study highlights the feasibility of identifying clinically relevant tumor antigens with direct immunopeptidomic profiling and supports their utilization as vaccines for moderately immunogenic cancer types. Furthermore, we present a pipeline integrating antigen discovery with a therapeutic cancer vaccine platform for improved personalized oncolytic cancer immunotherapy. Our dataset provides tools for further proof-of-concept testing of tumor antigen vaccination protocols in combination with other immunotherapies.

Supplementary Materials: The following are available online at https:/ /www.mdpi.com/article/10 .3390 / cancers13143408/s1, Figure S1: (A) Overal workflow for the discovery of MHC-I presented antigens. Tumor associated antigens were identified by searching the mouse reference proteome and ERV antigen by extending the search to include all TrEMBL entries. (B) Bioinformatic analysis workflow. (C) Gibbs clustering for the identified 10 mers and 11 mers and known H-2Kd and H-2Dd ligand binding motifs based on eluted, naturally presented ligand data., Figure S2: Individual tumor growth curves. Dotted lines represent threshold for responders and non-responders. The responder and non-responder mice are highlighted in green and red, respectively, and the percentage of responding mice within the group is given. $N=8$ for each group, Figure S3: Analysis of tumor infiltrating CD8+ T-cells. (A) Correlation analysis of tumor size at endpoint (day 21) vs. percentage of tumor infiltrating CD8+ cells. (B) Percentage of PD-1 positive CD8+ cells in tumors, Figure S4: Comparison of the vaccination platforms for stimulation of antigen-specific T-cells, Figure S5: Individual tumor growth curves for the therapeutic vaccination experiments using PeptiCRAd platform, Supplementary File S1 and S2: Additional data generated during this study are included in this published article as additional information files.

Author Contributions: K.P., R.M.M.B., J.L. and V.C. designed the study. K.P., R.M.M.B. and E.Y. set-up and optimized methodology. K.P., S.F., H.M.U., J.C., G.M., S.P. and R.M.M.B. performed experiments and related data analysis. All authors interpreted results. K.P. wrote the original manuscript draft. J.L. and V.C. supervised the study. J.L. and V.C. equally contributed as last author. All authors have read and agreed to the published version of the manuscript.

Funding: This study was financially supported by grants from European Research Council under the Horizon 2020 framework ERC-consolidator Grant (GA n. 681219), Jane and Aatos Erkko Foundation (project n. 4705796), HiLIFE Fellow (project n. 797011004), Cancer Finnish Foundation (project n. 4706116), Magnus Ehrnrooth Foundation (project n. 4706235), the Swedish Research Council (2015-04622), the Swedish Foundation for Strategic Research, Swedish Cancer Society, the Swedish Childhood Cancer Foundation, the Cancer Research Funds of Radiumhemmet.

Institutional Review Board Statement: The animal experiment was approved by the Experimental Animal Committee of Southern Finland National Animal Experiment Board, ELLA (license number ESAVI/9817/04.10.07/2016) and was performed under the guidelines of the of the Regional State Administrative Agencies in Finland.

Informed Consent Statement: Not applicable.

Data Availability Statement: The mass spectrometric raw datasets generated during the current study are available in the ProteomeXchange Consortium via the PRIDE partner repository with the dataset identifier PXD016112.

Acknowledgments: "Open access funding provided by University of Helsinki". The animal experiment was carried out at the Laboratory Animal Center (LAC), University of Helsinki, and the flow cytometric analysis was performed at the HiLife Flow Cytometry Unit at the University of Helsinki. We are grateful to Mikaela Grönholm for the scientific discussions and critical reading of the manuscript and Valo Therapeutics for providing their VALO-mD901 oncolytic virus for the study. 
Conflicts of Interest: V.C. is co-founder and shareholder in Valo Therapeutics LTD.

\section{References}

1. Lehmann, B.; Bauer, J.A.; Chen, X.; Sanders, M.E.; Chakravarthy, A.B.; Shyr, Y.; Pietenpol, J.A. Identification of human triplenegative breast cancer subtypes and preclinical models for selection of targeted therapies. J. Clin. Investig. 2011, 121, $2750-2767$. [CrossRef]

2. Liu, S.; Lachapelle, J.; Leung, S.; Gao, D.; Foulkes, W.D.; O Nielsen, T. CD8+ lymphocyte infiltration is an independent favorable prognostic indicator in basal-like breast cancer. Breast Cancer Res. 2012, 14, R48. [CrossRef] [PubMed]

3. Loi, S.; Sirtaine, N.; Piette, F.; Salgado, R.; Viale, G.; Van Eenoo, F.; Rouas, G.; Francis, P.; Crown, J.P.A.; Hitre, E.; et al. Prognostic and predictive value of tumor-infiltrating lymphocytes in a phase III randomized adjuvant breast cancer trial in node-positive breast cancer comparing the addition of docetaxel to doxorubicin with doxorubicin-based chemotherapy: BIG 02-98. J. Clin. Oncol. 2013, 31, 860-867. [CrossRef]

4. $\quad$ Emens, L.; Molinero, L.; Loi, S.; Rugo, H.S.; Schneeweiss, A.; Diéras, V.; Iwata, H.; Barrios, C.H.; Nechaeva, M.; Nguyen-Duc, A.; et al. Atezolizumab and nab-Paclitaxel in Advanced Triple-Negative Breast Cancer: Biomarker Evaluation of the IMpassion130 Study. J. Natl. Cancer Inst. 2021, 379, 2108-2121.

5. Mittendorf, E.A.; Philips, A.V.; Meric-Bernstam, F.; Qiao, N.; Wu, Y.; Harrington, S.; Su, X.; Wang, Y.; Gonzalez-Angulo, A.M.; Akcakanat, A.; et al. PD-L1 Expression in Triple-Negative Breast Cancer. Cancer Immunol. Res. 2014, 2, 361-370. [CrossRef]

6. Granados, D.P.; Laumont, C.M.; Thibault, P.; Perreault, C. The nature of self for T cells-A systems-level perspective. Curr. Opin. Immunol. 2015, 34, 1-8. [CrossRef] [PubMed]

7. Haen, S.; Rammensee, H.-G. The repertoire of human tumor-associated epitopes-Identification and selection of antigens and their application in clinical trials. Curr. Opin. Immunol. 2013, 25, 277-283. [CrossRef]

8. Hilf, N.; Kuttruff-Coqui, S.; Frenzel, K.; Bukur, V.; Stevanović, S.; Gouttefangeas, C.; Platten, M.; Tabatabai, G.; Dutoit, V.; Van Der Burg, S.H.; et al. Actively personalized vaccination trial for newly diagnosed glioblastoma. Nature 2019, 565, 240-245. [CrossRef]

9. Solovyov, A.; Vabret, N.; Arora, K.S.; Snyder, A.; Funt, S.A.; Bajorin, D.F.; Rosenberg, J.E.; Bhardwaj, N.; Ting, D.T.; Greenbaum, B.D. Global cancer transcriptome quantifies repeat element polarization between immunotherapy responsive and $\mathrm{T}$ cell suppressive classes. Cell Rep. 2018, 23, 512-521. [CrossRef]

10. Chiappinelli, K.B.; Strissel, P.L.; Desrichard, A.; Li, H.; Henke, C.; Akman, B.; Hein, A.; Rote, N.S.; Cope, L.M.; Snyder, A.; et al. Inhibiting DNA Methylation Causes an Interferon Response in Cancer via dsRNA Including Endogenous Retroviruses. Cell 2015, 162, 974-986. [CrossRef]

11. Saini, S.K.; Ørskov, A.D.; Bjerregaard, A.-M.; Unnikrishnan, A.; Holmberg-Thydén, S.; Borch, A.; Jensen, K.V.; Anande, G.; Bentzen, A.K.; Marquard, A.M.; et al. Human endogenous retroviruses form a reservoir of T cell targets in hematological cancers. Nat. Commun. 2020, 11, 5660. [CrossRef]

12. Bassani-Sternberg, M. Mass spectrometry based immunopeptidomics for the discovery of cancer neoantigens. Methods Mol. Biol. 2018, 1719, 209-221.

13. Kim, S.; Pevzner, P.A. MS-GF+ makes progress towards a universal database search tool for proteomics. Nat. Commun. 2014, 5, 5277. [CrossRef]

14. Andreatta, M.; Alvarez, B.; Nielsen, M. GibbsCluster: Unsupervised clustering and alignment of peptide sequences. Nucleic Acids Res. 2017, 45, W458-W463. [CrossRef] [PubMed]

15. Rapin, N.P.J.-P.; Hoof, I.; Lund, O.; Nielsen, M. MHC motif viewer. Immunogenetics 2008, 60, 759-765. [CrossRef] [PubMed]

16. Mi, H.; Muruganujan, A.; Ebert, D.; Huang, X.; Thomas, P.D. PANTHER version 14: More genomes, a new PANTHER GO-slim and improvements in enrichment analysis tools. Nucleic Acids Res. 2019, 47, D419-D426. [CrossRef] [PubMed]

17. Ylösmäki, E.; Ylösmäki, L.; Fusciello, M.; Martins, B.; Ahokas, P.; Cojoc, H.; Uoti, A.; Feola, S.; Kreutzman, A.; Ranki, T.; et al. Characterization of a novel OX40 ligand and CD40 ligand-expressing oncolytic adenovirus used in the PeptiCRAd cancer vaccine platform. Mol. Ther. Oncolytics 2021, 20, 459-469. [CrossRef] [PubMed]

18. Capasso, C.; Hirvinen, M.; Garofalo, M.; Romaniuk, D.; Kuryk, L.; Sarvela, T.; Vitale, A.; Antopolsky, M.; Magarkar, A.; Viitala, T.; et al. Oncolytic adenoviruses coated with MHC-I tumor epitopes increase the antitumor immunity and efficacy against melanoma. OncoImmunology 2016, 5, e1105429. [CrossRef]

19. Yadav, M.; Jhunjhunwala, S.; Phung, Q.T.; Lupardus, P.J.; Tanguay, J.; Bumbaca, S.; Franci, C.; Cheung, T.K.; Fritsche, J.; Weinschenk, T.; et al. Predicting immunogenic tumour mutations by combining mass spectrometry and exome sequencing. Nat. Cell Biol. 2014, 515, 572-576. [CrossRef]

20. Schuster, H.; Shao, W.; Weiss, T.; Pedrioli, P.G.; Roth, P.; Weller, M.; Campbell, D.S.; Deutsch, E.W.; Moritz, R.L.; Planz, O.; et al. A tissue-based draft map of the murine MHC class I immunopeptidome. Sci. Data 2018, 5, 180157. [CrossRef]

21. Hanahan, D.; Weinberg, R.A. The hallmarks of cancer. Cell 2000, 100, 57-70. [CrossRef]

22. Hanahan, D.; Weinberg, R.A. Hallmarks of cancer: The next generation. Cell 2011, 144, 646-674. [CrossRef] [PubMed]

23. Johansson, H.J.; Consortia Oslo Breast Cancer Research Consortium (OSBREAC); Socciarelli, F.; Vacanti, N.M.; Haugen, M.H.; Zhu, Y.; Siavelis, I.; Fernandez, A.; Aure, M.R.; Sennblad, B.; et al. Breast cancer quantitative proteome and proteogenomic landscape. Nat. Commun. 2019, 10,1-14. [CrossRef] [PubMed] 
24. Smith, C.C.; Beckermann, K.E.; Bortone, D.S.; De Cubas, A.A.; Bixby, L.M.; Lee, S.J.; Panda, A.; Ganesan, S.; Bhanot, G.; Wallen, E.M.; et al. Endogenous retroviral signatures predict immunotherapy response in clear cell renal cell carcinoma. J. Clin. Investig. 2018, 128, 4804-4820. [CrossRef]

25. Chiaro, J.; Kasanen, H.H.E.; Whalley, T.; Capasso, C.; Gronholm, M.; Feola, S.; Peltonen, K.; Hamdan, F.; Hemberg, M.; Mäkelä, S.; et al. Viral Molecular Mimicry Influences the Antitumor Immune Response in Murine and Human Melanoma. Cancer Immunol. Res. 2021. [CrossRef]

26. Blackburn, S.D.; Shin, H.; Haining, W.N.; Zou, T.; Workman, C.J.; Polley, A.; Betts, M.R.; Freeman, G.J.; Vignali, D.; Wherry, E.J. Coregulation of CD8+ T cell exhaustion by multiple inhibitory receptors during chronic viral infection. Nat. Immunol. 2008, 10, 29-37. [CrossRef]

27. Jin, H.-T.; Anderson, A.C.; Tan, W.G.; West, E.E.; Ha, S.-J.; Araki, K.; Freeman, G.J.; Kuchroo, V.K.; Ahmed, R. Cooperation of Tim-3 and PD-1 in CD8 T-cell exhaustion during chronic viral infection. Proc. Natl. Acad. Sci. USA 2010, 107, 14733-14738. [CrossRef]

28. Ahmadzadeh, M.; Johnson, L.A.; Heemskerk, B.; Wunderlich, J.R.; Dudley, M.E.; White, D.E.; Rosenberg, S.A. Tumor antigenspecific CD8 T cells infiltrating the tumor express high levels of PD-1 and are functionally impaired. Blood 2009, 114, 1537-1544. [CrossRef]

29. Baitsch, L.; Baumgaertner, P.; Devêvre, E.; Raghav, S.K.; Legat, A.; Barba, L.; Wieckowski, S.; Bouzourene, H.; Deplancke, B.; Romero, P.; et al. Exhaustion of tumor-specific CD8 ${ }^{+}$T cells in metastases from melanoma patients. J. Clin. Investig. 2011, 121, 2350-2360. [CrossRef]

30. Gros, A.; Robbins, P.F.; Yao, X.; Li, Y.F.; Turcotte, S.; Tran, E.; Wunderlich, J.R.; Mixon, A.; Farid, S.; Dudley, M.E.; et al. PD-1 identifies the patient-specific CD8+ tumor-reactive repertoire infiltrating human tumors. J. Clin. Investig. 2014, 124, 2246-2259. [CrossRef]

31. Gros, A.; Parkhurst, M.R.; Tran, E.; Pasetto, A.; Robbins, P.F.; Ilyas, S.; Prickett, T.D.; Gartner, J.J.; Crystal, J.S.; Roberts, I.M.; et al. Prospective identification of neoantigen-specific lymphocytes in the peripheral blood of melanoma patients. Nat. Med. 2016, 22, 433-438. [CrossRef] [PubMed]

32. Bourgeois-Daigneault, M.-C.; Roy, D.G.; Aitken, A.S.; El Sayes, N.; Martin, N.T.; Varette, O.; Falls, T.; St-Germain, L.; Pelin, A.; Lichty, B.D.; et al. Neoadjuvant oncolytic virotherapy before surgery sensitizes triple-negative breast cancer to immune checkpoint therapy. Sci. Transl. Med. 2018, 10, eaao1641. [CrossRef] [PubMed]

33. Feola, S.; Capasso, C.; Fusciello, M.; Martins, B.; Tähtinen, S.; Medeot, M.; Carpi, S.; Frascaro, F.; Ylosmäki, E.; Peltonen, K.; et al. Oncolytic vaccines increase the response to PD-L1 blockade in immunogenic and poorly immunogenic tumors. OncoImmunology 2018, 7, e1457596. [CrossRef] [PubMed]

34. Strausberg, R.L.; Feingold, E.A.; Grouse, L.H.; Derge, J.G.; Klausner, R.D.; Collins, F.S.; Wagner, L.; Shenmen, C.M.; Schuler, G.D.; Altschul, S.F.; et al. Generation and initial analysis of more than 15,000 full-length human and mouse cDNA sequences. Proc. Natl. Acad. Sci. USA 2002, 99, 16899-16903. [PubMed]

35. Ternette, N.; Olde Nordkamp, M.J.M.; Muller, J.; Anderson, A.P.; Nicastri, A.; Hill, A.V.S.; Kessler, B.M.; Li, D. Immunopeptidomic profiling of HLA-A2-positive triple negative breast cancer identifies potential immunotherapy target antigens. Proteomics 2018, 18, e1700465. [CrossRef] [PubMed]

36. Walter, S.; Weinschenk, T.; Stenzl, A.; Zdrojowy, R.; Pluzanska, A.; Szczylik, C.; Staehler, M.; Brugger, W.; Dietrich, P.-Y.; Mendrzyk, R.; et al. Multipeptide immune response to cancer vaccine IMA901 after single-dose cyclophosphamide associates with longer patient survival. Nat. Med. 2012, 18, 1254-1261. [CrossRef] [PubMed]

37. Takahashi, R.; Toh, U.; Iwakuma, N.; Takenaka, M.; Otsuka, H.; Furukawa, M.; Fujii, T.; Seki, N.; Kawahara, A.; Kage, M.; et al. Feasibility study of personalized peptide vaccination for metastatic recurrent triple-negative breast cancer patients. Breast Cancer Res. 2014, 16, R70. [CrossRef]

38. Zhao, Q.; Laverdure, J.-P.; Lanoix, J.; Durette, C.; Côté, C.; Bonneil, É.; Laumont, C.M.; Gendron, P.; Vincent, K.; Courcelles, M.; et al. Proteogenomics Uncovers a Vast Repertoire of Shared Tumor-Specific Antigens in Ovarian Cancer. Cancer Immunol. Res. 2020, 8, 544-555. [CrossRef]

39. Liu, J.; Blake, S.J.; Yong, M.C.R.; Harjunpää, H.; Ngiow, S.F.; Takeda, K.; Young, A.; O’Donnell, J.S.; Allen, S.; Smyth, M.J.; et al. Improved efficacy of neoadjuvant compared to adjuvant immunotherapy to eradicate metastatic disease. Cancer Discov. 2016, 6, 1382-1399. [CrossRef]

40. Burugu, S.; Gao, D.; Leung, S.; Chia, S.K.; Nielsen, T.O. TIM-3 expression in breast cancer. OncoImmunology 2018, 7, e1502128. [CrossRef] 